\title{
dCache
}

\section{a storage system for the future}

\author{
Patrick Fuhrmann
}

Volker Gülzow

for the dCache people

Euro-Par 2006 : European Conference On Parallel Computing 


\section{dCache, The project}

\section{(Preliminaries)}


Responsibility, dCache

Patrick Fuhrmann Rob Kennedy

Core Team (Desy and Fermi)

Jon Bakken

Ted Hesselroth

Alex Kulyavtsev

Birgit Lewendel

Dmitri Litvintsev

Tigran Mrktchyan

Martin Radicke

Owen Synge

Vladimir Podstavkov

\section{Responsibility, SRM}

Timur Perelmutov

\section{External}

Development

Nicolo Fioretti, BARI, Italy

Abhishek Singh Rana, SDSC, US

Support and Help

Maarten Lithmaath, CERN

N.N, CERN 


\section{Related Projects}

\section{*Fermilab}

\section{dCache}

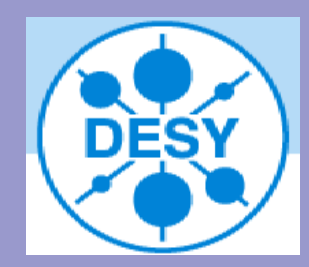

(C) SciDAC D

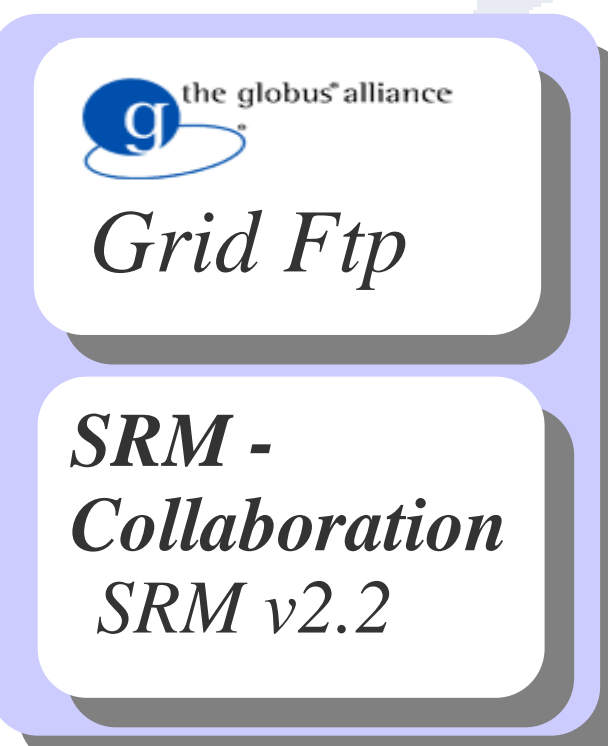

\section{$\neq \mathrm{UCSD}$}

$$
\text { gPlazma }
$$

Grid based authorization

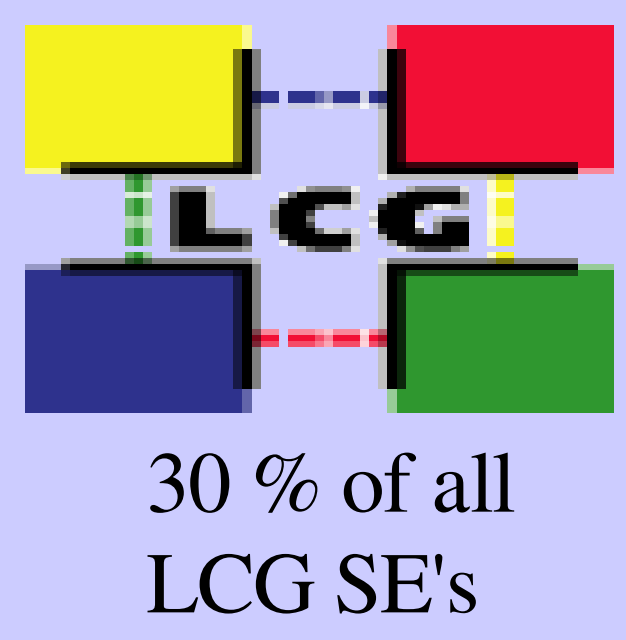

Open Science Grid

Mainly the large SE's in OSG

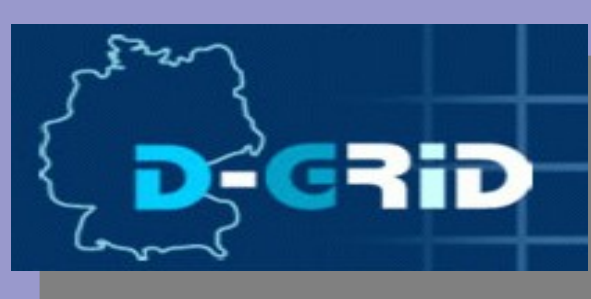

HEPCG Project

Scalable Storage Element

Coscheduling

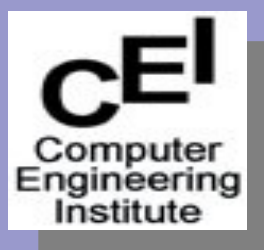

Integration Project (DGI)

Core Grid Middleware

@ Jiilich (FZJ/ZAM) 


\section{Zoom into Grid Storage}

Fast Zoom into $L C G$

Grid Enabled Managed Storage 


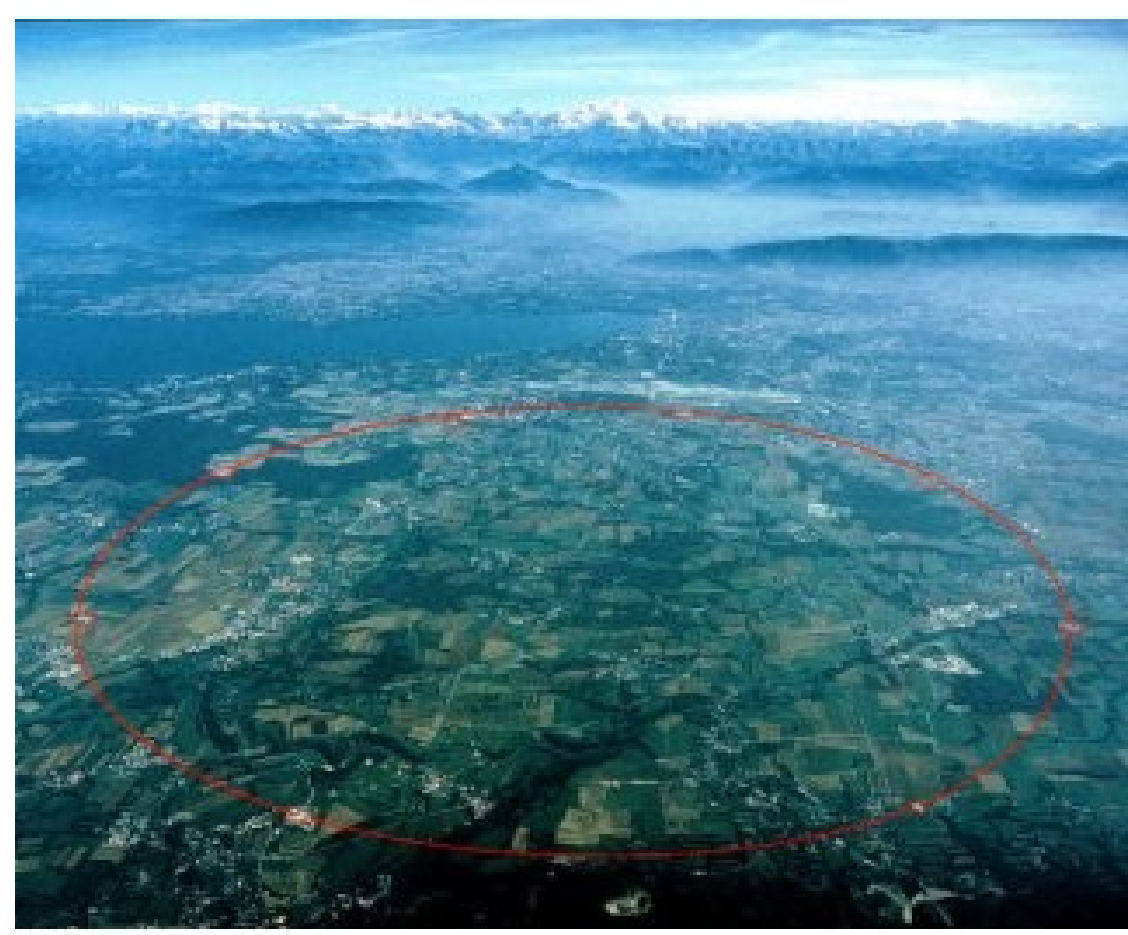

$1 \mathrm{TeV}$ is about the energy of motion of a flying mosquito. What makes the LHC so extraordinary is that it squeezes energy into a space about a million million times smaller than a mosquito. (Stolen from CERN Web Pages)
$27 \mathrm{Km}$ super conducting double ring Proton collisions 20 events / collision $14 \mathrm{TeV}$ center of mass

$300 \mathrm{Mbytes} / \mathrm{second}$ raw data output

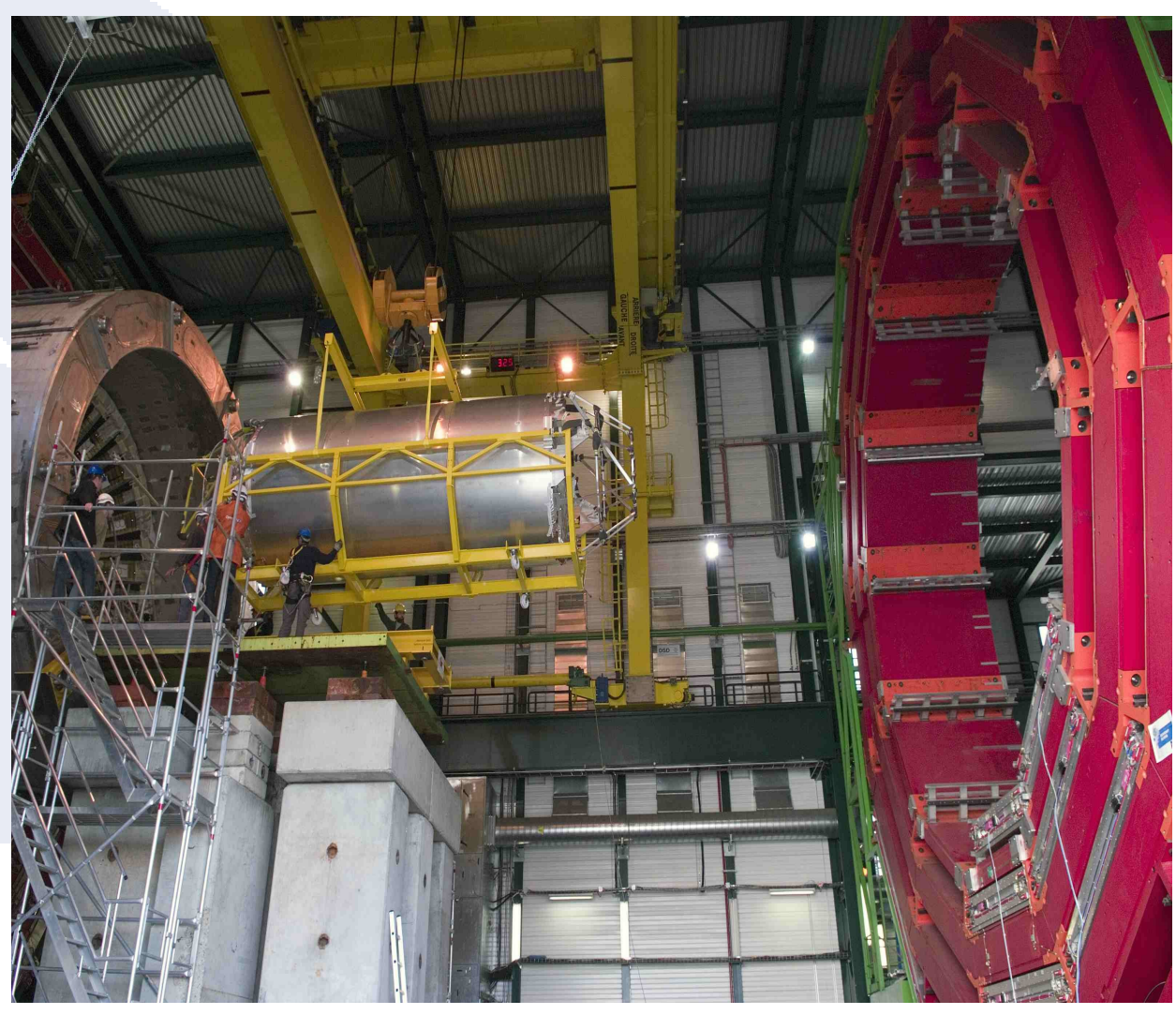




\section{LCG Tier approach}

Tier 0-2 Storage Elements (SE)

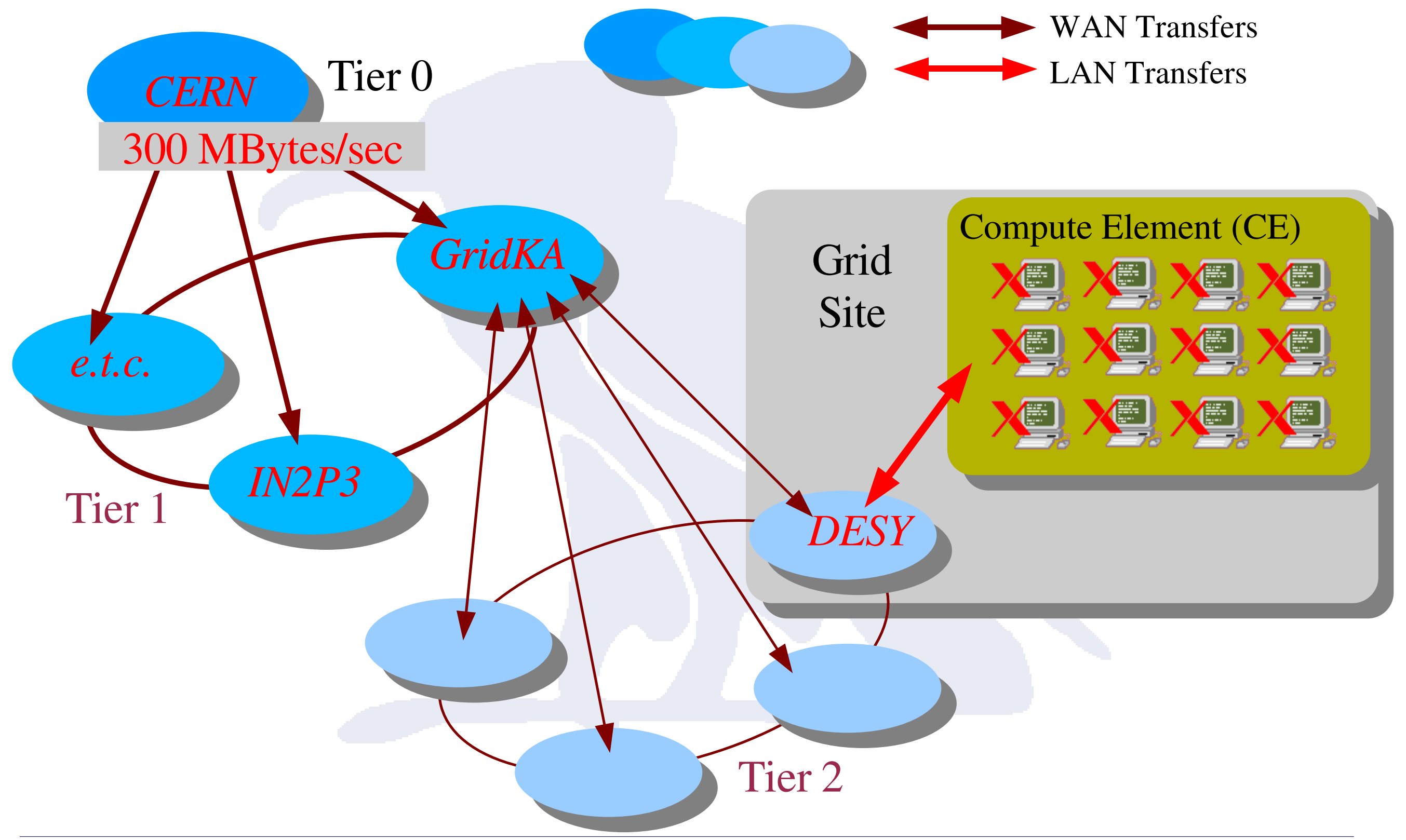




\section{Information Provider Details}

by courtesy of Nicolò Fioretti

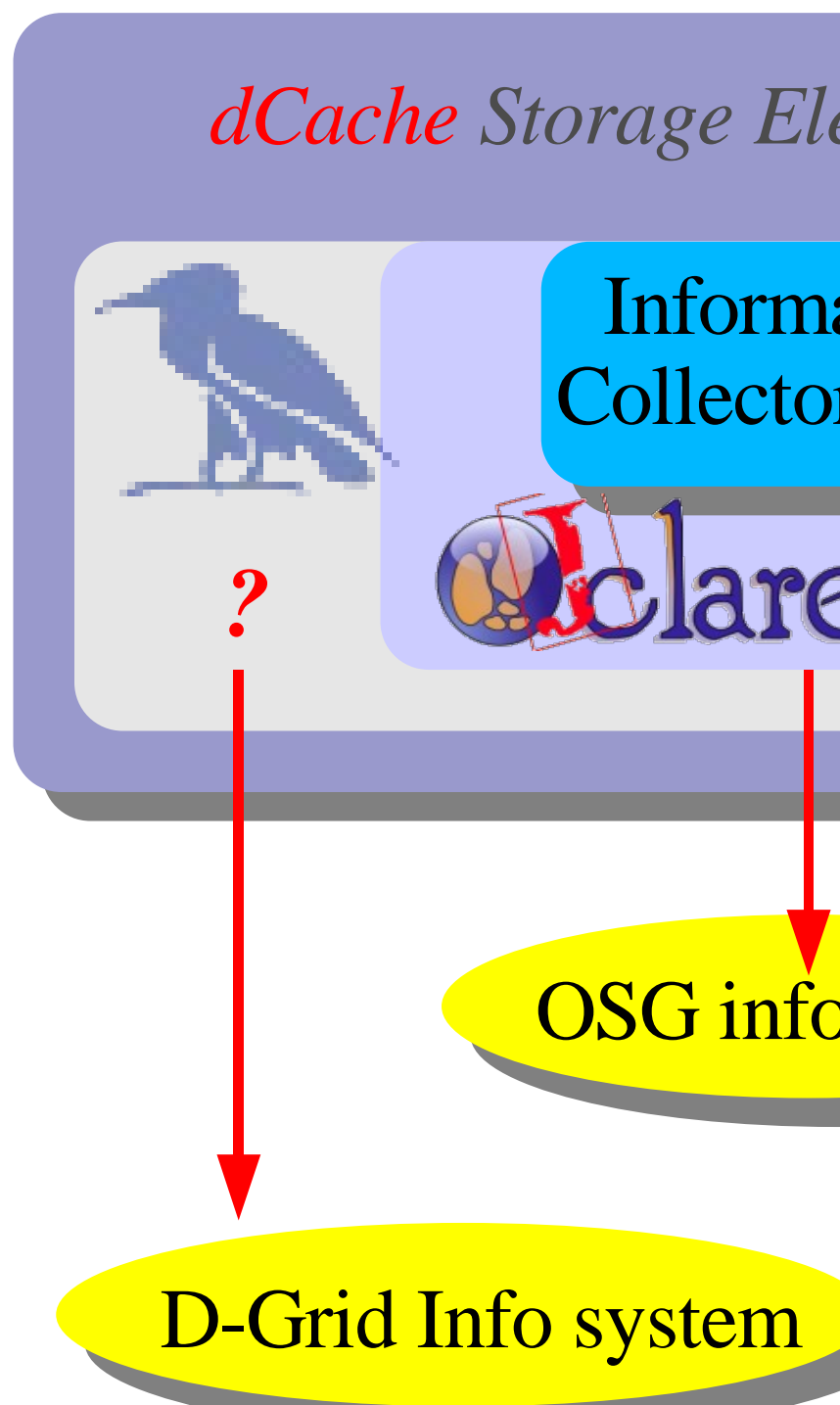

dynamic information

Glue schema 2.1

including Space

Information per $\mathrm{VO}$ static information

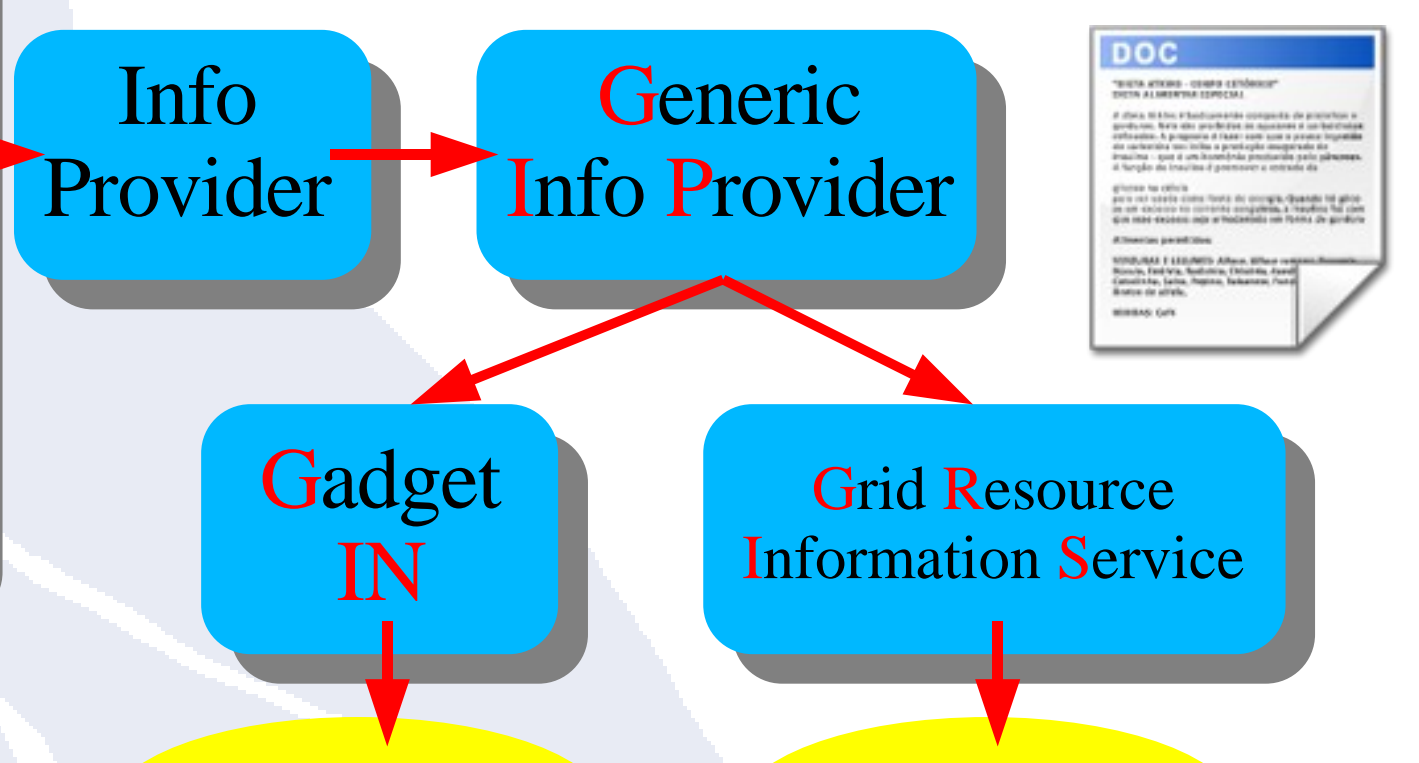

\section{R-GMA}

\section{MDS}

with many thanks to Jean-Philippe Baud and Lawrence Field

CHEP06 : A computational and data scheduling architecture for HEP applications by Martin Radicke and Lars Schley 


\section{The SRM Interface definition}

\section{SRM is a Storage Management Abstraction}

From the SRM project pages (sdm.lbl.gov/srm-wg/index.html) :

This is an international collaboration among CERN,FNAL, JLAB, LBNL and RAL.

From the SRM fermi pages (srm.fnal.gov) :

SRM is the Storage Resource Manager layer providing storage and location independent access to data.

Technically :

- Prepares for data transfer (not transfer itself) by storage URL (SRUL)

- Negotiates data transfer protocol (theoretically).

- May initiate restore of data from back-end storage systems.

- Delivers 'transfer url' (TURL) for subsequent transfer (gsiFtp,httpg).

- Supports directory functions including file listings.

- Supports space reservation functionality (implicit and explicit via space tokens)

- Supports 'property spaces' :

File Properties resp. Property classes Media Quality Persistence probability of data loss permanent $* * *$ volatile
Availability

how long does it take to get this file ready for $\mathrm{I} / \mathrm{O}$
Tape0 Disk1

$\longrightarrow$ Tape1 Disk0

Tape1 Disk1 


\section{Storage Elements}

\section{currently available}




\section{CASTOR}

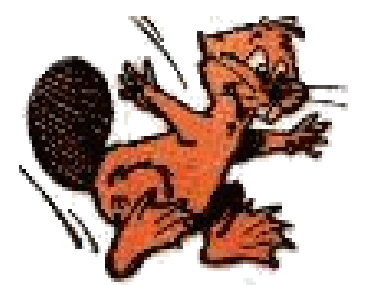

Developed at CERN; CERN's main repository

HSM included

For huge installations only

Support available but requires man power compensation to CERN

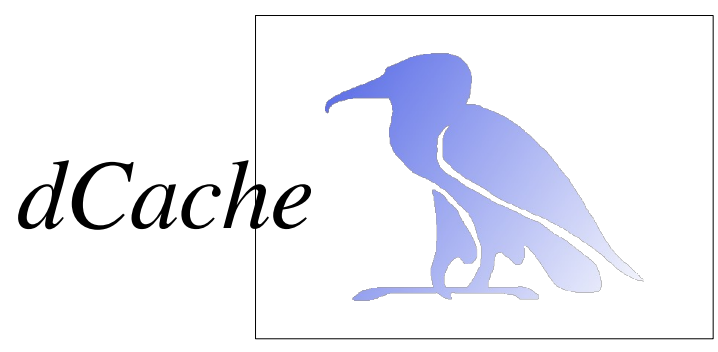

Developed at DESY/FERMI can talk to many HSM's

From small to huge installations ( $>200$ Tbytes on disk)

Support for free ( support@dCache.ORG)

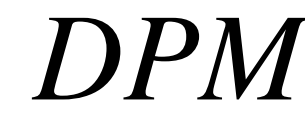

Disk Pool Manager

Developed at CERN

for HSM less installations only

From small to medium installations

Support not clear 


\section{Berkeley DRM}

Developed and supported by LBL (Lawrence Berkeley National Laboratory)

Used by Open science grid (OSG)

Part of VDT (Virtual Data Toolkit, Globus)

\section{Non Storage Element (SRM Interface only)}

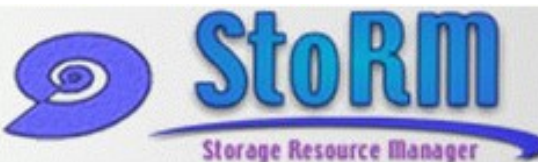

StoRM
Developed at INFN, CNAF, Italy Independent SRM implementation Interacts with regular filesystem Enhanced support for GPFS (space reservation) 


\section{Insight}

\section{dCache}




\section{dCache is Managed Storage}

Distributed Peta Byte Disk Store with single rooted file-system providing posix like and wide area access protocols.

Distributed cache system to optimize access to Tertiary Storage Systems

Grid Storage Element coming with standard data access protocols, Information Provider Protocols and Storage Resource Manager. 


\section{Basic Specification}

Single 'rooted' file system name space tree

Strict separation between data and name-space

File system names space view available through an nfs $2 / 3$ interface

Data is distributed among a huge amount of disk servers.

Supports multiple internal and external copies of a single file

Supports 'posix like' access (dCap, xRoot) as well as various FTP dialects, (http) and the Storage Resource Manager Protocol. 
File system view

File content view

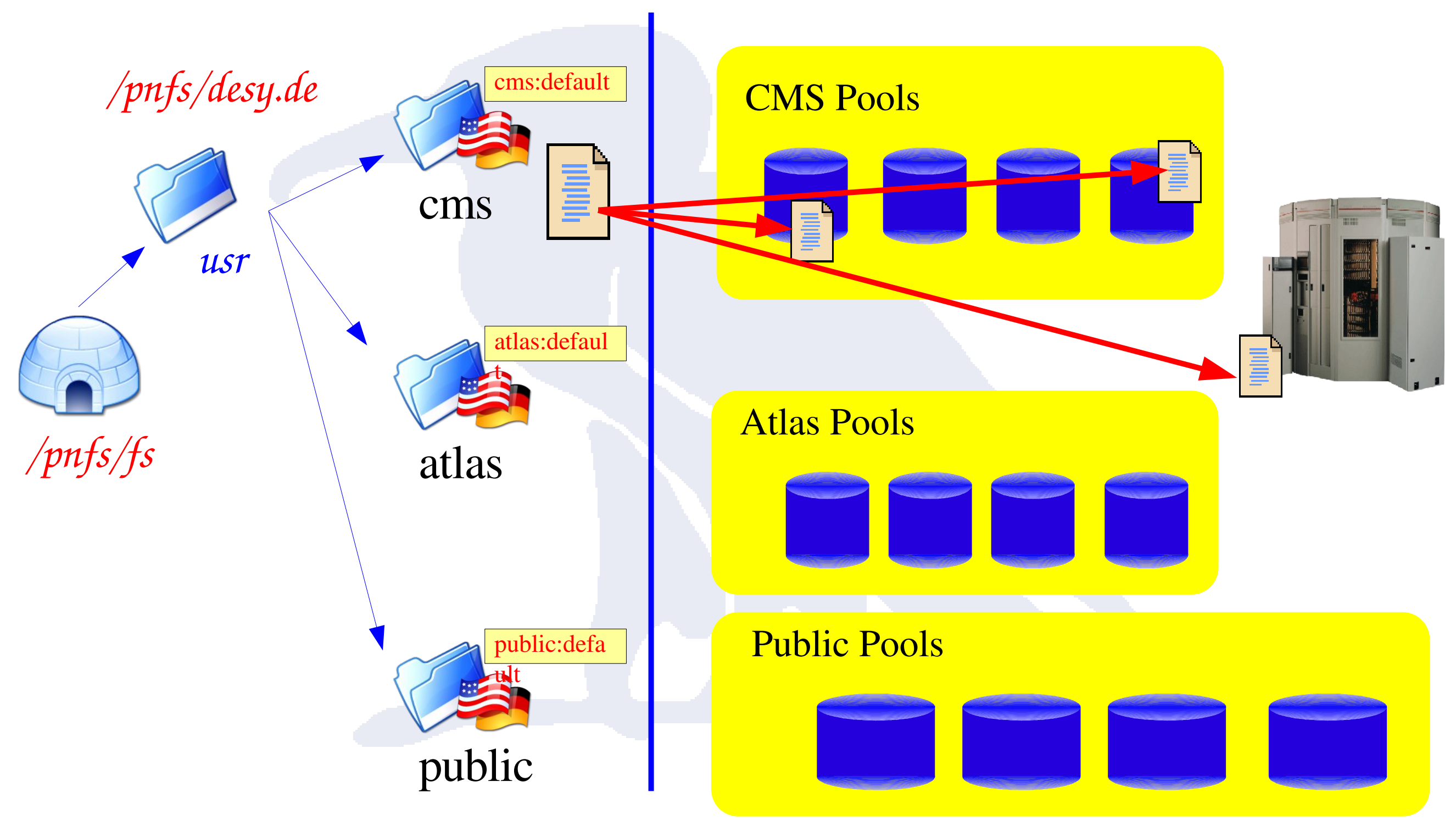


SRM for storage management

$n f s 2 / 3$ for name space

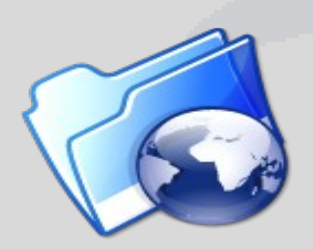

/pnfs/ $<$ site $\rangle /<\mathrm{VO}\rangle / \ldots$

dCap, $x$ Root for random LAN

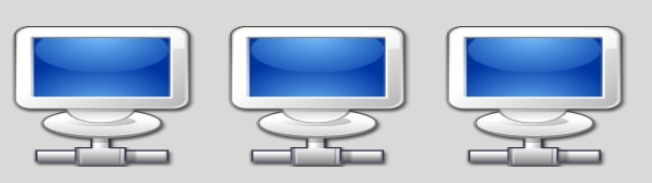

gsiFtp, $h t t p(g)$ for random WAN

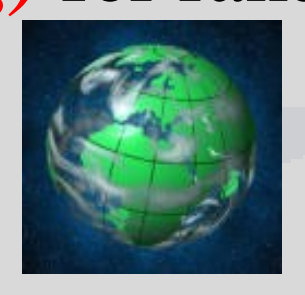

dCache

Osm, Enstore, Tsm, Hpss, ... 


\section{Selected}

Topics 


\section{File hopping I}

- Automatic data set replication on hot spot detection.

- File replication on client read request (pools disallowed for reading)

- Dataset replication on arriving of datasets. 


\section{Internal distribution of incoming data dCache.ORG}

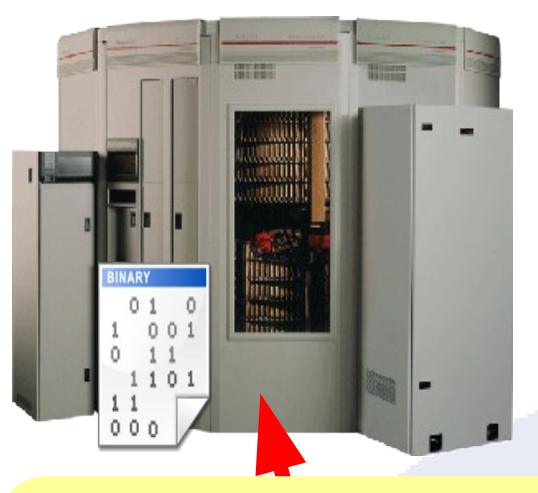

Collect and flush

\section{Write Only|Cache}
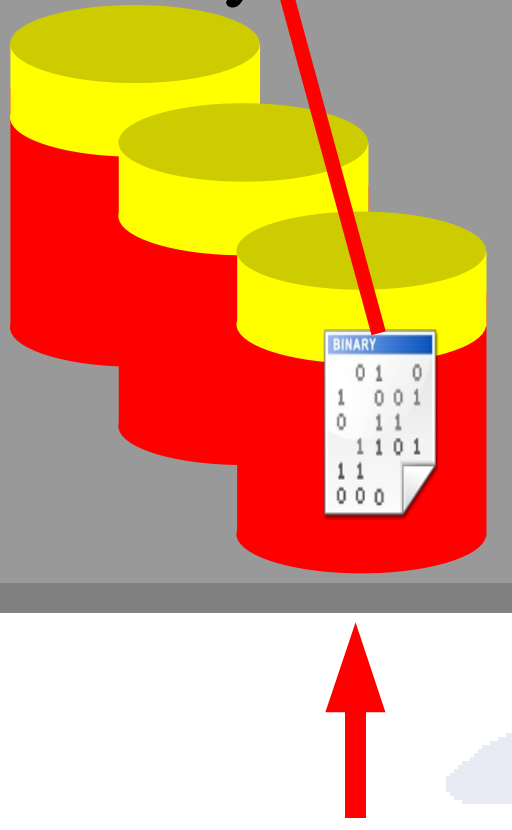

\section{Read Only Cache}

Forward on read or on arrival
To Client

From Client 


\section{HSM interactions}

- Datasets collected in write pools and flushed according to rules.

- Centrally controlled (Smart) flushing -> ping pong

- Datasets restored if requested but no longer in cache.

- Intermediate restore pool for HSM optimization. 


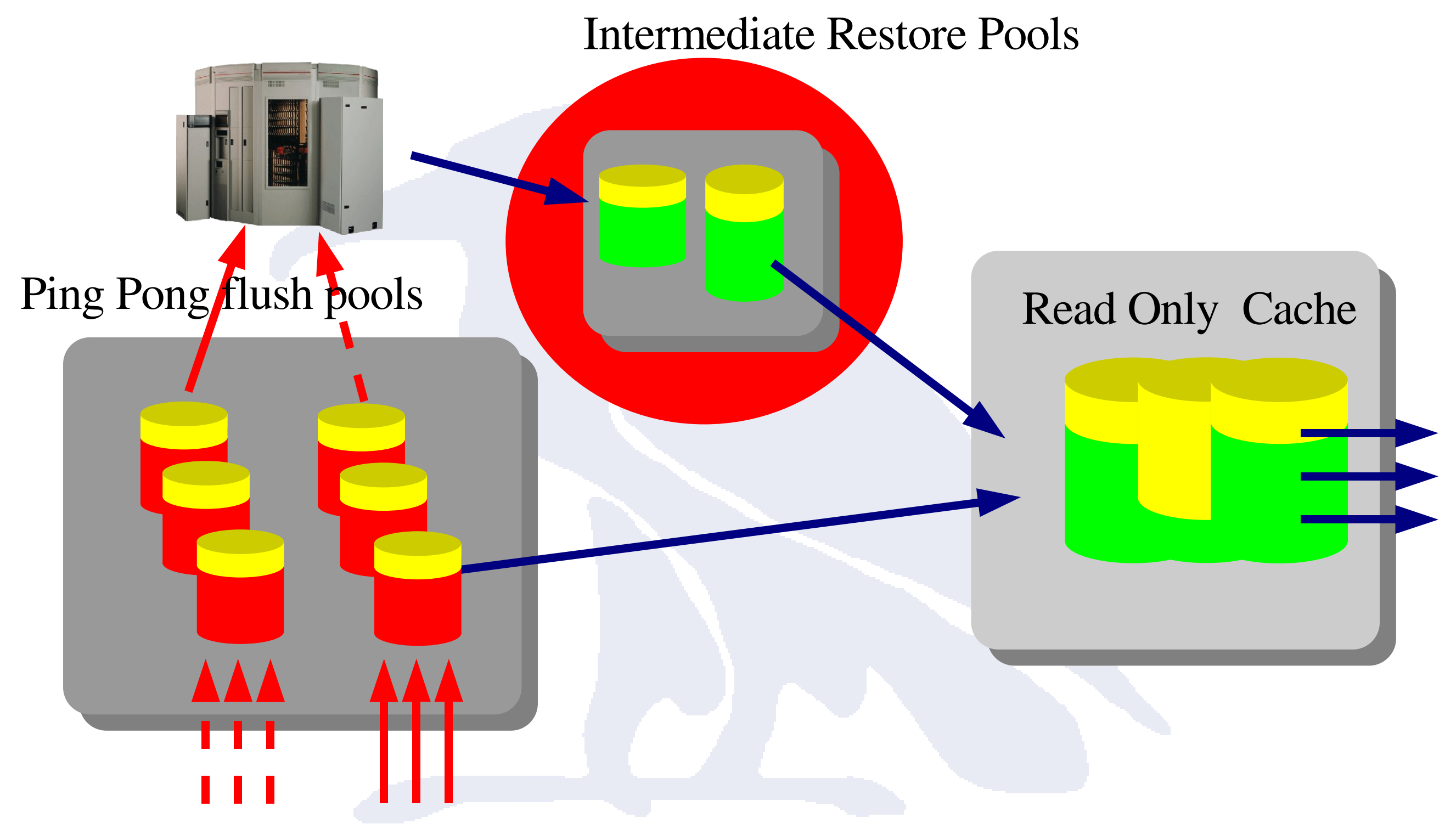

From Client 


\section{N Nearly all hoppings dCache.ORG}

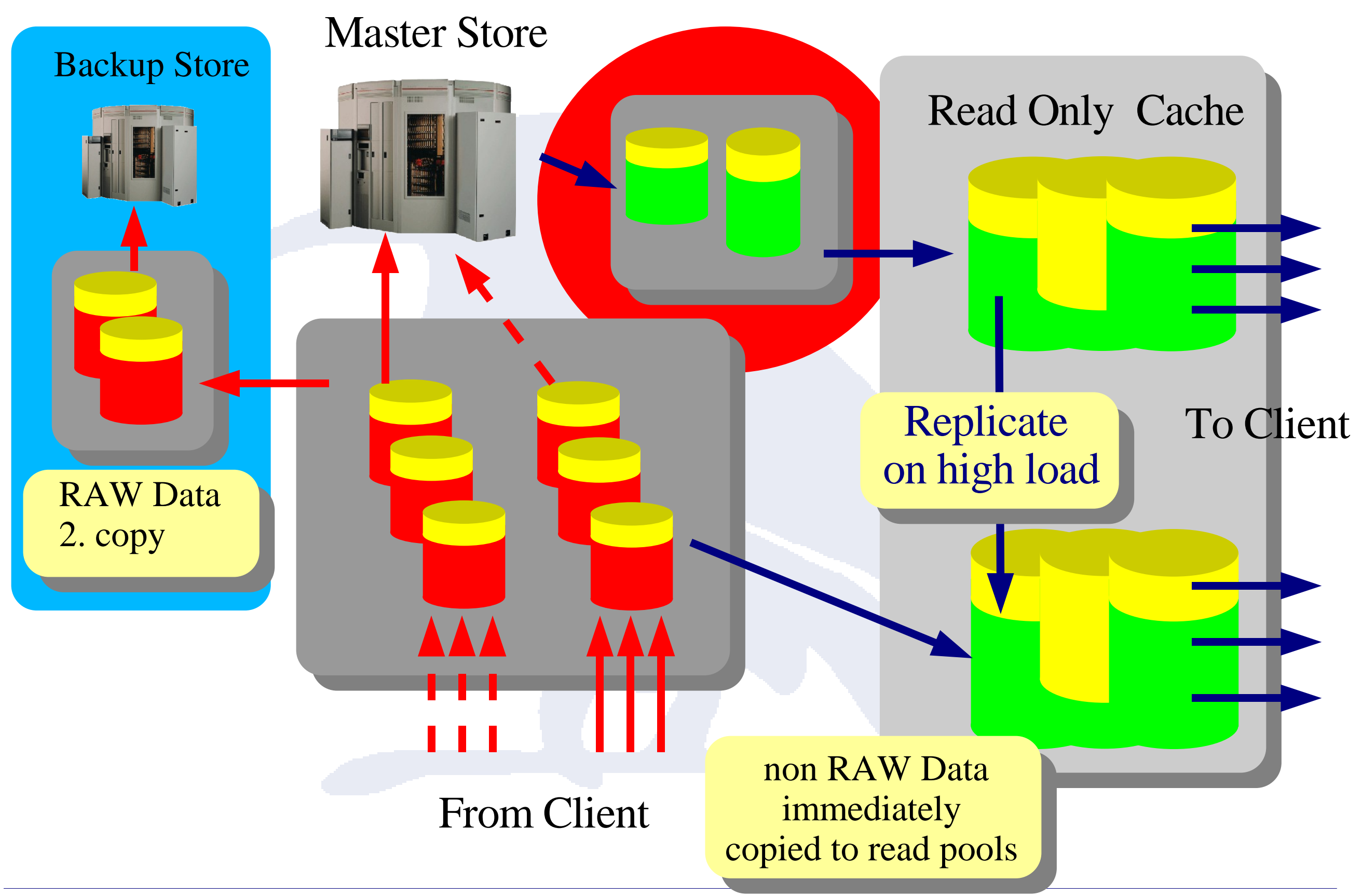




\section{And now for something completely different $d$ Cache. $O R G$}

By courtesy of Alexander Kulyavtsev

\section{Resilient dCache (pools on worker nodes)}

- Controls number of copies for each dataset in dCache

- Makes sure $\mathrm{n}<$ copies $<\mathrm{m}$

- Adjusts replica count on pool failures

- Adjusts replica count on scheduled pool maintenance

\section{Attractive because :}

- $\mathrm{N}$ - pool nodes may be in maintenance mode without affecting the overall availability of datasets in the dCache system.

- Improves overall performance by read striping

- Makes use of unused space on worker/farm nodes. 


\section{And not to forget ...}

- Destination pool selection by IP, directory, protocol, I/O direction.

- Final pool selection by space cost and pool node load.

- dCache instance partitioning.

- Extended proxy (certificate) support (OSG and LCG)

- Draining of pools for maintenance.

- Rich command line interface (via ssh).

- First version of GUI for admin and cpu/space cost analysis.

- Highly improved file system emulation (chimera) in evaluation phase.

- See 'dCache, the Book' for details. 
Speed

Number of 'opens' per second

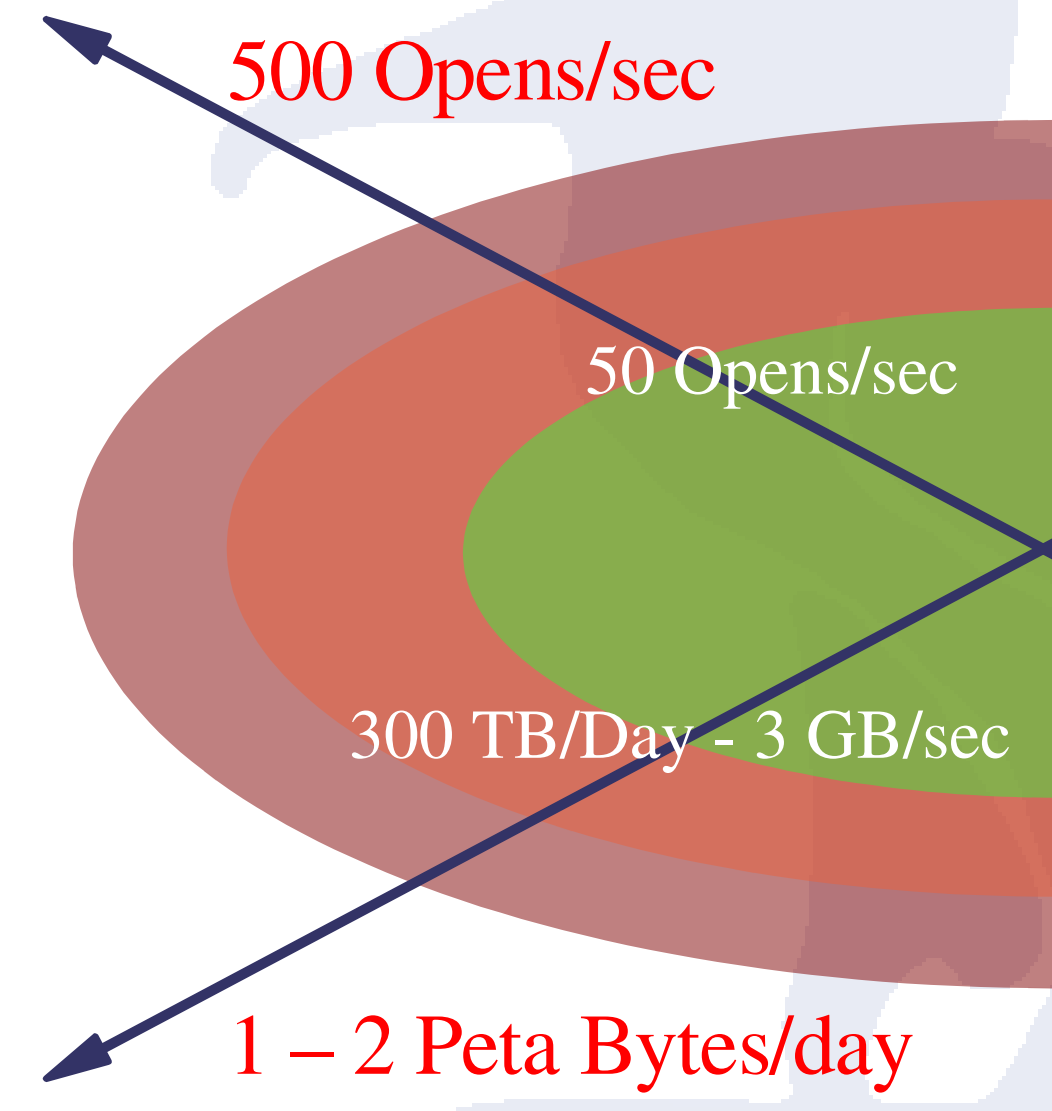

Number of bytes per second
Storage Space

Number of pools

\section{0 pools}

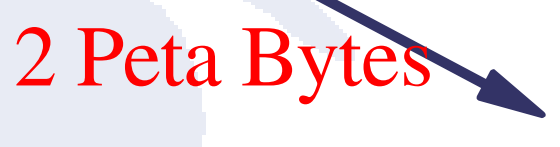




\section{Tier I centers :}

FNAL, BNL, US

IN2P3, France

SARA, Nl

Nordu Grid, Sweden, Denmark, Norway ...

gridKa, Karlruhe, Gr

RAL, UK

Triump, Canada

\section{Tier II centers :}

Germany

LCG : Aachen, DESY, Freiburg, Dortmund,Darmstadt(GSI)

Italy

d-Grid : Juelich(ZAM), Berlin(ZIB)

UK

$30 \%$ of gridPP, UK

INFN : Bari, Torino

Poland, Bulgaria, Spain

Canada, Taiwan

US

CMS : 7 sites, ATLAS 7 sites in preparation 
dCache, the Book

\section{www.dCache.ORG}

need specific help for you installation or help

in designing your dCache instance.

$$
\text { support@dCache.ORG }
$$

$\mathrm{dCache}$ user forum

$$
\text { user-forum@dCache.ORG }
$$

